\title{
A Note on Saigo's Fractional Integral Inequalities
}

\author{
Guotao Wang ${ }^{1, *}$, Harshvardhan Harsh ${ }^{2}$, S.D. Purohit ${ }^{3}$, Trilok Gupta ${ }^{4}$ \\ ${ }^{1}$ School of Mathematics and Computer Science, Shanxi Normal University, Linfen, Shanxi, People's Republic of China \\ ${ }^{2}$ Department of Mathematics, Amity University, Jaipur, India \\ ${ }^{3}$ Department of Basic Sciences (Mathematics), College of Technology and Engineering, M.P. University of Agriculture and \\ Technology, Udaipur, India \\ ${ }^{4}$ Department of Civil Engineering, College of Technology and Engineering, M.P. University of Agriculture and Technology, Udaipur, \\ India \\ *Corresponding author:.wgt2512@163.com
}

Received May 01, 2014; Revised June 03, 2014; Accepted June 12, 2014

\begin{abstract}
In this paper, some new integral inequalities related to the bounded functions, involving Saigo's fractional integral operators, are eshtablished. Special cases of the main results are also pointed out.
\end{abstract}

Keywords: Integral inequalities, Gauss hypergeometric function, Saigo's fractional integral operators

Cite This Article: Guotao Wang, Harshvardhan Harsh, S.D. Purohit, and Trilok Gupta, "A Note on Saigo's Fractional Integral Inequalities." Turkish Journal of Analysis and Number Theory, vol. 2, no. 3 (2014): 65-69. doi: 10.12691/tjant-2-3-2.

\section{Introduction and Preliminaries}

Under various assumptions (Chebyshev inequality, Grüss inequality, Minkowski inequality, Hermite- Hadamard inequality, Ostrowski inequality etc.), inequalities are playing a very significant role in all fields of mathematics, particularly in the theory of approximations (see $[2,6,7,13,14,17,23]$ ). Therefore, in the literature we found several extensions and generalizations of these integral inequalities for the functions of bounded variation, synchronous, Lipschitzian, monotonic, absolutely continuous and n-times differentiable mappings etc. ([10,11,12,15,16,19,20,21,22,26,27,28]). In the past recent years, one more dimension have been added to this study, by introducing number of integral inequalities involving various fractional calculus and qcalculus operators. For detailed account, one may refer $[1,3,4,5,8,9,18,24,25,29-35]$ and the references cited therein.

Recently, Tariboon et al. [33] investigated certain new integral inequalities for the integrable functions, whose bounds are also integrable functions, involving the Riemann-Liouville fractional integral operators. Our aim in this paper, is to obtain a general extensions of the results due to Tariboon et al. [33]. Main results investigated here provide certain new integral inequalities associated with the integrable functions, whose bounds are also integrable functions, involving the Saigo's fractional integral operators. We also give some consequent results and special cases of the main results.

Firstly, we mention below the basic definitions and notations of some well-known operators of fractional calculus, which shall be used in the sequel.

Let $\alpha>0, \beta, \eta \in \mathbb{R}$, then the Saigo fractional integral $I_{0, t}^{\alpha, \beta, \eta}$ of order $\alpha$ for a real-valued continuous function $f(t)$ is defined by ([36], see also [[37], p. 19]):

$$
I_{0, t}^{\alpha, \beta, \eta} f(t)=\frac{t^{-\alpha-\beta}}{\Gamma(\alpha)} \int_{0}^{t}{ }_{2} F_{1}\left(\alpha+\beta,-\eta ; \alpha ; 1-\frac{\tau}{t}\right) f(\tau){ }^{\alpha \tau,(1.1)}
$$

where, the function ${ }_{2} F_{1}(-)$ appearing as a kernel for the operator (1.1) is the Gaussian hypergeometric function defined by

$$
{ }_{2} F_{1}(a, b ; c ; t)=\sum_{n=0}^{\infty} \frac{(a)_{n}(b)_{n}}{(c)_{n}} \frac{t^{n}}{n !},
$$

and $(a)_{n}$ is the Pochhammer symbol

$$
(a)_{n}=a(a+1) \ldots(a+n-1),(a)_{0}=1 .
$$

The operator $I_{0, t}^{\alpha, \beta, \eta}$ includes both the RiemannLiouville and the Erdélyi-Kober fractional integral operators given by the following relationships:

$$
\begin{aligned}
I^{\alpha} f(t) & =I_{0, t}^{\alpha,-\alpha, 0} f(t) \\
& =\frac{1}{\Gamma(\alpha)} \int_{0}^{t}(t-\tau)^{\alpha-1} f(\tau) d \tau(\alpha>0)
\end{aligned}
$$

and

$$
\begin{aligned}
& I^{\alpha, \eta} f(t)=I_{0, t}^{\alpha, 0, \eta} f(t) \\
& =\frac{t^{-\alpha-\eta}}{\Gamma(\alpha)} \int_{0}^{t}(t-\tau)^{\alpha-1} \tau^{n} f(\tau) d \tau(\alpha>0, \eta \in \mathbb{R}) .
\end{aligned}
$$

Following [36], for $f(t)=t^{\mu}$ in (1.1), we get

$$
\begin{aligned}
& I_{0, t}^{\alpha, \beta, \eta} t^{\mu}=\frac{\Gamma(\mu+1) \Gamma(\mu+1-\beta+\eta)}{\Gamma(\mu+1-\beta) \Gamma(\mu+1+\alpha+\eta)} t^{\mu-\beta} \\
& (\alpha>0, \min (\mu, \mu-\beta+\eta)>-1, t>0) .
\end{aligned}
$$




\section{Main Results}

In this section, we obtain certain integral inequalities, related to the integrable functions, whose bounds are also integrable functions, involving Saigo's fractional hypergeometric operators. The results are given in the form of the following theorems:

Theorem 1. Let $f, \varphi_{1}$, and $\varphi_{2}$ are integrable functions defined on $[0, \infty)$, such that

$$
\varphi_{1}(t) \leq f(t) \leq \varphi_{2}(t), \text { for all } t \in[0, \infty) .
$$

Then, for $t>0$, we have

$$
\begin{aligned}
& I_{0, t}^{\gamma, \delta, \zeta} \varphi_{1}(t) I_{0, t}^{\alpha, \beta, \eta} f(t)+I_{0, t}^{\alpha, \beta, \eta} \varphi_{2}(t) I_{0, t}^{\gamma, \delta, \zeta} f(t) \\
\geq & I_{0, t}^{\alpha, \beta, \eta} \varphi_{2}(t) I_{0, t}^{\gamma, \delta, \zeta} \varphi_{1}(t)+I_{0, t}^{\alpha, \beta, \eta} f(t) I_{0, t}^{\gamma, \delta, \zeta} f(t),
\end{aligned}
$$

where $\alpha>\max \{0,-\beta\}, \beta<1, \beta-1<\eta<0, \gamma>\max \{0,-\delta\}$, $\delta<1$ and $\delta-1<\zeta<0$.

Proof. By the hypothesis of inequality (2.1), for any $\tau, \rho>0$, we have

$$
\left(\varphi_{2}(\tau)-f(\tau)\right)\left(f(\rho)-\varphi_{1}(\rho)\right) \geq 0,
$$

which follows that

$$
\begin{gathered}
\varphi_{2}(\tau) f(\rho)+\varphi_{1}(\rho) f(\tau) \\
\geq \varphi_{1}(\rho) \varphi_{2}(\tau)+f(\tau) f(\rho) .
\end{gathered}
$$

Consider

$$
\begin{aligned}
& F(t, \tau)=\frac{t^{-\alpha-\beta}(t-\tau)^{\alpha-1}}{\Gamma(\alpha)}{ }_{2} F_{1}\left(\alpha+\beta,-\eta ; \alpha ; 1-\frac{\tau}{t}\right) \\
& (\tau \in(0, t) ; t>0) \\
& =\frac{1}{\Gamma(\alpha)} \frac{(t-\tau)^{\alpha-1}}{t^{\alpha+\beta}}+\frac{(\alpha+\beta)(-\eta)}{\Gamma(\alpha+1)} \frac{(t-\tau)^{\alpha}}{t^{\alpha+\beta+1}} \\
& +\frac{(\alpha+\beta)(\alpha+\beta+1)(-\eta)(-\eta+1)}{2 \Gamma(\alpha+2)} \frac{(t-\tau)^{\alpha+1}}{t^{\alpha+\beta+2}}+\ldots,
\end{aligned}
$$

which remains positive, for all $\tau \in(0, t)(t>0)$, under the conditions stated with Theorem 1. Multiplying both sides of (2.3) by $F(t, \tau)$ (where $F(t, \tau)$ is given by (2.4)) and integrating the resulting identity with respect to $\tau$ from 0 to $t$, and using (1.1), we get

$$
\begin{aligned}
& f(\rho) I_{0, t}^{\alpha, \beta, \eta} \varphi_{2}(t)+\varphi_{1}(\rho) I_{0, t}^{\alpha, \beta, \eta} f(t) \\
\geq & \varphi_{1}(\rho) I_{0, t}^{\alpha, \beta, \eta} \varphi_{2}(t)+f(\rho) I_{0, t}^{\alpha, \beta, \eta} f(t) .
\end{aligned}
$$

Next, on multiplying both sides of (2.5) by

$$
\begin{aligned}
H(t, \rho) & =\frac{t^{-\gamma-\delta}(t-\rho)^{\gamma-1}}{\Gamma(\gamma)}{ }_{2} F_{1}(\gamma+\delta,-\zeta ; \gamma ; 1-\rho) \\
(\rho \in(0, t) ; t>0) &
\end{aligned}
$$

which also remains positive, for all $\rho \in(0, t)(t>0)$. Upon integrating the resulting inequality so obtained with respect to $\rho$ from 0 to $t$, and using the operator (1.1), we easily arrive at the desired result (2.1).
It may be noted that, for $\gamma=\alpha, \delta=\beta, \zeta=\eta$, the Theorem 1 immediately reduces to the following result:

Corollary 1. Let $\varphi_{1}$ and $\varphi_{2}$ are integrable functions defined on $[0, \infty)$ and satisfying inequality (2.1). Then, for $t>0$, we have

$$
\begin{aligned}
& I_{0, t}^{\alpha, \beta, \eta} \varphi_{1}(t) I_{0, t}^{\alpha, \beta, \eta} f(t)+I_{0, t}^{\alpha, \beta, \eta} \varphi_{2}(t) I_{0, t}^{\alpha, \beta, \eta} f(t) \\
\geq & I_{0, t}^{\alpha, \beta, \eta} \varphi_{2}(t) I_{0, t}^{\alpha, \beta, \eta} \varphi_{1}(t)+\left(I_{0, t}^{\alpha, \beta, \eta} f(t)\right),
\end{aligned}
$$

where $\alpha>\max \{0,-\beta\}, \beta<1$ and $\beta-1<\eta<0$.

Theorem 2. Let $f$ and $g$ be two integrable functions on $[0, \infty)$ and $\varphi_{1}, \varphi_{2}, \psi_{1}$ and $\psi_{2}$ are four integrable functions on $[0, \infty)$, such that

$$
\begin{gathered}
\varphi_{1}(t) \leq f(t) \leq \varphi_{2}(t), \psi_{1}(t) \leq g(t) \leq \psi_{2}(t), \\
\text { for all } t \in[0, \infty) .
\end{gathered}
$$

Then, for $t>0, \alpha>\max \{0,-\beta\}, \beta<1, \beta-1<\eta<0$, $\gamma>\max \{0,-\delta\}, \delta<1$ and $\delta-1<\zeta<0$, the following inequalities holds true:

$$
\begin{gathered}
I_{0, t}^{\gamma, \delta, \zeta} \psi_{1}(t) I_{0, t}^{\alpha, \beta, \eta} f(t)+I_{0, t}^{\alpha, \beta, \eta} \varphi_{2}(t) I_{0, t}^{\gamma, \delta, \zeta} g(t) \\
\geq I_{0, t}^{\alpha, \beta, \eta} \varphi_{2}(t) I_{0, t}^{\gamma, \delta, \zeta} \psi_{1}(t)+I_{0, t}^{\alpha, \beta, \eta} f(t) I_{0, t}^{\gamma, \delta, \zeta} g(t), \\
I_{0, t}^{\gamma, \delta, \zeta} \varphi_{1}(t) I_{0, t}^{\alpha, \beta, \eta} g(t)+I_{0, t}^{\alpha, \beta, \eta} \psi_{2}(t) I_{0, t}^{\gamma, \delta, \zeta} f(t) \\
\geq I_{0, t}^{\alpha, \beta, \eta} \psi_{2}(t) I_{0, t}^{\gamma, \delta, \zeta} \varphi_{1}(t)+I_{0, t}^{\alpha, \beta, \eta} g(t) I_{0, t}^{\gamma, \delta, \zeta} f(t), \\
I_{0, t}^{\gamma, \delta, \zeta} \varphi_{2}(t) I_{0, t}^{\alpha, \beta, \eta} \psi_{2}(t)+I_{0, t}^{\alpha, \beta, \eta} f(t) I_{0, t}^{\gamma, \delta, \zeta} g(t) \\
\geq I_{0, t}^{\alpha, \beta, \eta} \varphi_{2}(t) I_{0, t}^{\gamma, \delta, \zeta} g(t)+I_{0, t}^{\alpha, \beta, \eta} f(t) I_{0, t}^{\gamma, \delta, \zeta} \psi_{2}(t), \\
I_{0, t}^{\gamma, \delta, \zeta} \varphi_{1}(t) I_{0, t}^{\alpha, \beta, \eta} \psi_{1}(t)+I_{0, t}^{\alpha, \beta, \eta} f(t) I_{0, t}^{\gamma, \delta, \zeta} g(t) \\
\geq I_{0, t}^{\alpha, \beta, \eta} \varphi_{1}(t) I_{0, t}^{\gamma, \delta, \zeta} f(t)+I_{0, t}^{\alpha, \beta, \eta} f(t) I_{0, t}^{\gamma, \delta, \zeta} \psi_{1}(t) .
\end{gathered}
$$

Proof. Let $f$ and $g$ are two integrable functions and satisfying inequality (2.8), then to prove (2.9), we can write

$$
\left(\varphi_{2}(\tau)-f(\tau)\right)\left(g(\rho)-\psi_{1}(\rho)\right) \geq 0,
$$

which follows that

$$
\begin{gathered}
\varphi_{2}(\tau) g(\rho)+\psi_{1}(\rho) f(\tau) \\
\geq \psi_{1}(\rho) \varphi_{2}(\tau)+f(\tau) g(\rho) .
\end{gathered}
$$

On multiplying both sides of (2.13) by $F(t, \tau)$ (where $F(t, \tau)$ is given by (2.4)) and integrating with respect to $\tau$ from 0 to $t$, then by making use of (1.1), we get

$$
\begin{aligned}
& g(\rho) I_{0, t}^{\alpha, \beta, \eta} \varphi_{2}(t)+\psi_{1}(\rho) I_{0, t}^{\alpha, \beta, \eta} f(t) \\
\geq & \psi_{1}(\rho) I_{0, t}^{\alpha, \beta, \eta} \varphi_{2}(t)+g(\rho) I_{0, t}^{\alpha, \beta, \eta} f(t) .
\end{aligned}
$$

Next, multiplying both sides of (2.13) by $H(t, \rho)(\rho \in(0, t), t>0)$, where $H(t, \rho)$ is given by (2.6), and integrating with respect to $\rho$ from 0 to $t$, we easily arrive at the desired result (2.9). 
Following the similar procedure, one can easily establish the remaining inequalities (2.10) to (2.12) by using the following inequalities, respectively

$$
\begin{aligned}
& \left(\psi_{2}(\tau)-g(\tau)\right)\left(f(\rho)-\varphi_{1}(\rho)\right) \geq 0, \\
& \left(\varphi_{2}(\tau)-f(\tau)\right)\left(g(\rho)-\psi_{2}(\rho)\right) \geq 0
\end{aligned}
$$

and

$$
\left(\varphi_{1}(\tau)-f(\tau)\right)\left(g(\rho)-\psi_{1}(\rho)\right) \geq 0 .
$$

Therefore, we omit the further details of the proof of these results.

\section{Consequent Results and Special Cases}

The Saigo's fractional integral operator defined by (1.1), possess the advantage that the Erdélyi-Kober and the Riemann-Liouville type fractional integral operators happen to be the particular cases of this operator. Therefore, by suitably specializing the parameters, we now briefly consider some special cases of the result derived in the preceding section. To this end, let us set $\beta=0$ and $\delta=0$, and make use of the relation (1.4), then Theorems 1 \& 2 yields the following inequalities involving the Erdélyi-Kober type fractional integral operators:

Corollary 2. Let $f, \varphi$ and $\varphi_{2}$ are integrable functions defined on $[0, \infty)$ and satisfying inequality (2.1), then for $t>0$, we have

$$
\begin{gathered}
I^{\gamma, \zeta} \varphi_{1}(t) I^{\alpha, \eta} f(t)+I^{\alpha, \eta} \varphi_{2}(t) I^{\gamma, \zeta} f(t) \\
\geq I^{\alpha, \eta} \varphi_{2}(t) I^{\gamma, \zeta} \varphi_{1}(t)+I^{\alpha, \eta} f(t) I^{\gamma, \zeta} f(t),
\end{gathered}
$$

where $\alpha>0,-1<\eta<0, \gamma>0$ and $-1<\zeta<0$.

Corollary 3. Let $f$ and $g$ be two integrable functions on $[0, \infty)$ and $\varphi_{1}, \varphi_{2}, \psi_{1}$ and $\psi_{2}$ are four integrable functions on $[0, \infty)$, and satisfying inequality (2.8). Then, for $t>0, \alpha>0,-1<\eta<0, \gamma>0$ and $-1<\zeta<0$, the following inequalities holds true:

$$
\begin{aligned}
& I^{\gamma, \zeta} \psi_{1}(t) I^{\alpha, \eta} f(t)+I^{\alpha, \eta} \varphi_{2}(t) I^{\gamma, \zeta} g(t) \\
\geq & I^{\alpha, \eta} \varphi_{2}(t) I^{\gamma, \zeta} \psi_{1}(t)+I^{\alpha, \eta} f(t) I^{\gamma, \zeta} g(t), \\
& I^{\gamma, \zeta} \varphi_{1}(t) I^{\alpha, \eta} g(t)+I^{\alpha, \eta} \psi_{2}(t) I^{\gamma, \zeta} f(t) \\
\geq & I^{\alpha, \eta} \psi_{2}(t) I^{\gamma, \zeta} \varphi_{1}(t)+I^{\alpha, \eta} g(t) I^{\gamma, \zeta} f(t), \\
& I^{\gamma, \zeta} \varphi_{2}(t) I^{\alpha, \eta} \psi_{2}(t)+I^{\alpha, \eta} f(t) I^{\gamma, \zeta} g(t) \\
\geq & I^{\alpha, \eta} \varphi_{2}(t) I^{\gamma, \zeta} g(t)+I^{\alpha, \eta} f(t) I^{\gamma, \zeta} \psi_{2}(t), \\
& I^{\gamma, \zeta} \varphi_{1}(t) I^{\alpha, \eta} \psi_{1}(t)+I^{\alpha, \eta} f(t) I^{\gamma, \zeta} g(t) \\
\geq & I^{\alpha, \eta} \varphi_{1}(t) I^{\gamma, \zeta} f(t)+I^{\alpha, \eta} f(t) I^{\gamma, \zeta} \psi_{1}(t) .
\end{aligned}
$$

Next, if we replace $\beta$ by $-\alpha, \delta$ by $-\gamma$ and make use of the relation (1.3), then Theorems $1 \& 2$ corresponds to the known integral inequalities involving RiemannLiouville type fractional integral operators, due to Tariboon et al [33].
Further, if we put $\varphi_{1}(t)=m, \varphi_{2}(t)=M, \psi_{1}(t)=p$ and $\psi_{2}(t)=P$, where $m, M, p, P \in \mathbb{R}, \forall t \in[0, \infty)$ and make use of formula (1.5), then the Theorems $1 \& 2$ leads to the following particluar results:

Corollary 4. Let $f$ be an integrable function defined on $[0, \infty)$, such that

$$
m \leq f(t) \leq M, \quad m, M \in \mathbb{R} \text { for all } t \in[0, \infty) .
$$

Then, for $t>0$, we have

$$
\begin{aligned}
& m \frac{\Gamma(1-\delta+\zeta) t^{-\delta}}{\Gamma(1-\delta) \Gamma(1+\gamma+\zeta)} I_{0, t}^{\alpha, \beta, \eta} f(t) \\
+ & M \frac{\Gamma(1-\beta+\eta) t^{-\beta}}{\Gamma(1-\beta) \Gamma(1+\alpha+\eta)} I_{0, t}^{\gamma, \delta, \zeta} f(t) \\
\geq & m M \frac{\Gamma(1-\beta+\eta) \Gamma(1-\delta+\zeta) t^{-\beta-\delta}}{\Gamma(1-\beta) \Gamma(1+\alpha+\eta) \Gamma(1-\delta) \Gamma(1+\gamma+\zeta)} \\
+ & I_{0, t}^{\alpha, \beta, \eta} f(t) I_{0, t}^{\gamma, \delta, \zeta} f(t),
\end{aligned}
$$

where $\alpha>\max \{0,-\beta\}, \beta<1, \beta-1<\eta<0, \gamma>\max \{0,-\delta\}$, $\delta<1$ and $\delta-1<\zeta<0$.

Corollary 5. Let $f$ and $g$ be two integrable functions on $[0, \infty)$, such that

$$
\begin{aligned}
& m \leq f(t) \leq M, p \leq g(t) \leq P, \\
& m, p, M, P \in \mathbb{R} \text { for all } t \in[0, \infty) .
\end{aligned}
$$

Then, for $t>0, \alpha<\max \{0,-\beta\}, \beta<1, \quad \beta-1<\mu<0$, $\gamma>\max \{0,-\delta\}, \quad \delta<1$ and $\delta-1<\zeta<0$, the following inequalities holds true:

$$
\begin{aligned}
& p \frac{\Gamma(1-\delta+\zeta) t^{-\delta}}{\Gamma(1-\delta) \Gamma(1+\gamma+\zeta)} I_{0, t}^{\alpha, \beta, \eta} f(t) \\
+ & M \frac{\Gamma(1-\beta+\eta) t^{-\beta}}{\Gamma(1-\beta) \Gamma(1+\alpha+\eta)} I_{0, t}^{\gamma, \delta, \zeta} g(t) \\
\geq & p M \frac{\Gamma(1-\beta+\eta) \Gamma(1-\delta+\zeta) t^{-\beta-\delta}}{\Gamma(1-\beta) \Gamma(1+\alpha+\eta) \Gamma(1-\delta) \Gamma(1+\gamma+\zeta)} \\
+ & I_{0, t}^{\alpha, \beta, \eta} f(t) I_{0, t}^{\gamma, \delta, \zeta} g(t), \\
& m \frac{\Gamma(1-\delta+\zeta) t^{-\delta}}{\Gamma(1-\delta) \Gamma(1+\gamma+\zeta)} I_{0, t}^{\alpha, \beta, \eta} g(t) \\
+ & P \frac{\Gamma(1-\beta+\eta) t^{-\beta}}{\Gamma(1-\beta) \Gamma(1+\alpha+\eta)} I_{0, t}^{\gamma, \delta, \zeta} f(t) \\
\geq & m P \frac{\Gamma(1-\beta+\eta) \Gamma(1-\delta+\zeta) t^{-\beta-\delta}}{\Gamma(1-\beta) \Gamma(1+\alpha+\eta) \Gamma(1-\delta) \Gamma(1+\gamma+\zeta)} \\
+ & I_{0, t}^{\alpha, \beta, \eta} g(t) I_{0, t}^{\gamma, \delta, \zeta} f(t), \\
+ & I_{0, t}^{\alpha, \beta, \eta} f(t) I_{0, t}^{\gamma, \delta, \zeta} g(t) \\
& M P \frac{\Gamma(1-\beta+\eta) \Gamma(1-\delta+\zeta) t^{-\beta-\delta}}{\Gamma(1-\beta) \Gamma(1+\alpha+\eta) \Gamma(1-\delta) \Gamma(1+\gamma+\zeta)}
\end{aligned}
$$




$$
\begin{gathered}
\geq M \frac{\Gamma(1-\beta+\eta) t^{-\beta}}{\Gamma(1-\beta) \Gamma(1+\alpha+\eta)} I_{0, t}^{\gamma, \delta, \zeta} g(t) \\
+P \frac{\Gamma(1-\delta+\zeta) t^{-\delta}}{\Gamma(1-\delta) \Gamma(1+\gamma+\zeta)} I_{0, t}^{\alpha, \beta, \eta} f(t), \\
m p \frac{\Gamma(1-\beta+\eta) \Gamma(1-\delta+\zeta) t^{-\beta-\delta}}{\Gamma(1-\beta) \Gamma(1+\alpha+\eta) \Gamma(1-\delta) \Gamma(1+\gamma+\zeta)} \\
+I_{0, t}^{\alpha, \beta, \eta} f(t) I_{0, t}^{\gamma, \delta, \zeta} g(t) \\
\geq m \frac{\Gamma(1-\beta+\eta) t^{-\beta}}{\Gamma(1-\beta) \Gamma(1+\alpha+\eta)} I_{0, t}^{\gamma, \delta, \zeta} g(t) \\
+p \frac{\Gamma(1-\delta+\zeta) t^{-\delta}}{\Gamma(1-\delta) \Gamma(1+\gamma+\zeta)} I_{0, t}^{\alpha, \beta, \eta} f(t) .
\end{gathered}
$$

Again, if we set $\varphi_{1}(t)=t$ and $\varphi_{2}(t)=t+1$ and make use of formula (1.5), then the Theorem 1 and Corollary 1 , further leads to the following integral inequalities:

Corollary 6. Let $f$ be an integrable function defined on $[0, \infty)$, such that

$$
t \leq f(t) \leq t+1, \text { for all } t \in[0, \infty)
$$

Then, for $t>0, \alpha>\max \{0,-\beta\}, \beta<1, \beta-1<\eta<0$, $\gamma>\max \{0, \delta\}, \delta<1$ and $\delta-1<\zeta<0$ we have

$$
\begin{aligned}
& \frac{\Gamma(2-\delta+\zeta) t^{1-\delta}}{\Gamma(2-\delta) \Gamma(2+\gamma+\zeta)} I_{0, t}^{\alpha, \beta, \eta} f(t) \\
+ & \left(\begin{array}{c}
\frac{\Gamma(2-\beta+\eta) t^{1-\beta}}{\Gamma(2-\beta) \Gamma(2+\alpha+\eta)} \\
+\frac{\Gamma(1-\beta+\eta) t^{-\beta}}{\Gamma(1-\beta) \Gamma(1+\alpha+\eta)}
\end{array}\right) I_{0, t}^{\gamma, \delta, \zeta} f(t) \\
\geq & \left(\begin{array}{c}
\frac{\Gamma(2-\beta+\eta) t^{1-\beta}}{\Gamma(2-\beta) \Gamma(2+\alpha+\eta)} \\
+\frac{\Gamma(1-\beta+\eta) t^{-\beta}}{\Gamma(1-\beta) \Gamma(1+\alpha+\eta)}
\end{array}\right)\left(\frac{\Gamma(2-\delta+\zeta) t^{1-\delta}}{\Gamma(2-\delta) \Gamma(2+\gamma+\zeta)}\right) \\
+ & I_{0, t}^{\alpha, \beta, \eta} f(t) I_{0, t}^{\gamma, \delta, \zeta} f(t) .
\end{aligned}
$$

Corollary 7. Let $f$ be an integrable function defined on $[0, \infty)$, such that

$$
t \leq f(t) \leq t+1, \text { for all } t \in[0, \infty)
$$

Then, for $t>0, \alpha>\max \{0,-\beta\}, \beta<1$ and $\beta-1<\eta<0$, we have

$$
\begin{aligned}
& \left(\begin{array}{c}
\frac{2 \Gamma(2-\beta+\eta) t^{1-\beta}}{\Gamma(2-\beta) \Gamma(2+\alpha+\eta)} \\
+\frac{\Gamma(1-\beta+\eta) t^{-\beta}}{\Gamma(1-\beta) \Gamma(1+\alpha+\eta)}
\end{array}\right) I_{0, t}^{\alpha, \beta, \eta} f(t) \\
& \geq\left(I_{0, t}^{\alpha, \beta, \eta} f(t)\right)^{2} \\
& +\left(\begin{array}{c}
\frac{\Gamma(2-\beta+\eta) t^{1-\beta}}{\Gamma(2-\beta) \Gamma(2+\alpha+\eta)} \\
+\frac{\Gamma(1-\beta+\eta) t^{-\beta}}{\Gamma(1-\beta) \Gamma(1+\alpha+\eta)}
\end{array}\right) \times\left(\frac{\Gamma(2-\beta+\eta) t^{1-\beta}}{\Gamma(2-\beta) \Gamma(2+\alpha+\eta)}\right) .
\end{aligned}
$$

In this paper, we have introduced certain general integral inequalities, related to the integrable and bounded functions $f$ and $g$, involving Saigo's fractional integral operators. Therefore, we conclude with the remark that, by suitably specializing the arbitrary function $\varphi_{1}(t), \varphi_{2}(t)$, $\psi_{1}(t)$ and $\psi_{2}(t)$, one can further easily obtain additional integral inequalities involving the Riemann-Liouville, Erd'elyi-Kober and Saigo type fractional integral operators from our main results.

\section{Conflict of Interests}

The authors declare that there is no conflict of interests regarding the publication of this article.

\section{References}

[1] Anastassiou, G.A.: Advances on Fractional Inequalities, Springer Briefs in Mathematics, Springer, New York, 2011.

[2] Ahmadmir, N. and Ullah, R.: Some inequalities of Ostrowski and Gr" uss type for triple integrals on time scales, Tamkang J. Math., 42(4) (2011), 415-426.

[3] Baleanu, D. and Purohit, S.D.: Chebyshev type integral inequalities involving the fractional hypergeometric operators, Abstract Appl. Anal., 2014, Article ID 609160, 10 (pp).

[4] Baleanu, D., Purohit, S.D. and Agarwal, P.: On fractional integral inequalities involving hypergeometric operators, Chinese Journal of Mathematics, 2014, Article ID 609476, 5(pp.).

[5] Belarbi, S. and Dahmani, Z.: On some new fractional integral inequalities, J. Inequal. Pure Appl. Math., 10(3)(2009), Art. 86, 5 (pp).

[6] Cerone, P. and Dragomir, S.S.: New upper and lower bounds for the Chebyshev functional, J. Inequal. Pure App. Math., 3 (2002), Article 77.

[7] Chebyshev, P.L.: Sur les expressions approximatives des integrales definies par les autres prises entre les mêmes limites, Proc. Math. Soc. Charkov, 2(1882), 93-98.

[8] Dahmani, Z. and Benzidane, A.: New weighted Gruss type inequalities via $(\alpha, \beta)$ fractional qintegral inequalities, International Journal of Innovation and Applied Studies, 1(1)(2012), 76-83

[9] Dahmani, Z., Tabharit, L. and Taf, S.: New generalisations of Gr"uss inequality using Riemann- Liouville fractional integrals, Bull. Math. Anal. Appl., 2 (3)(2010), 93-99.

[10] Dragomir, S.S.: A generalization of Grüss's inequality in inner product spaces and applications, J. Math. Anal. Appl., 237 (1999), 74-82.

[11] Dragomir, S.S.: A Grüss type inequality for sequences of vectors in inner product spaces and applications, J. Inequal. Pure Appl. Math., 1(2) (2000), 1-11.

[12] Dragomir, S.S.: Some integral inequalities of Grüss type, Indian J. Pure Appl. Math., 31(4)(2000), 397-415.

[13] Dragomir, S.S.: Operator Inequalities of the Jensen,Čebyšev and Grüss Type, Springer Briefs in Mathematics, Springer, New York, 2012.

[14] Dragomir, S.S. and Wang, S.: An inequality of Ostrowski-Grüss' type and its applications to the estimation of error bounds for some special means and for some numerical quadrature rules, Comput. Math. Appl., 13(11) (1997), 15-20.

[15] Gauchman, H.: Integral inequalities in q-calculus, Comput. Math. Appl., 47 (2004), 281-300.

[16] Gavrea, B.: Improvement of some inequalities of Chebysev-Grüss type, Comput. Math. Appl., 64 (2012), 2003-2010.

[17] Grüss, D.: Uber das maximum des absoluten Betrages von $\frac{1}{b-a} \int_{a}^{b} f(x) g(x) d x-\frac{1}{(b-a)^{2}} \int_{a}^{b} f(x) d x \int_{a}^{b} g(x) d x$, Math. Z., 39(1935), 215-226. 
[18] Kalla, S.L. and Rao, Alka: On Grüss type inequality for hypergeometric fractional integrals, Le Matematiche, 66 (1)(2011), 57-64.

[19] Kapoor, G.: On some discrete Gruss type inequalities, Int Jr. of Mathematical Sciences \& Applications, 2(2) (2012), 729-734.

[20] Liu, Z.: Some Ostrowski-Grüss type inequalities and applications, Comput. Math. Appl., 53 (2007), 73-79.

[21] Maticć, M.: Improvment of some inequalities of Euler-Grüss type, Comput. Math. Appl., 46 (2003), 1325-1336.

[22] Mercer, McD A.: An improvement of the Grüss inequality, J. Inequa. Pure Appl. Math., 6(4) (2005), 1-4

[23] Mitrinović, D.S., Pečarić, J.E. and Fink, A.M.: Classical and New Inequalities in Analysis, Kluwer Academic, 1993.

[24] Ntouyas, S.K., Purohit, S.D. and Tariboon, J.: Certain Chebyshev type integral inequalities involving the Hadamard's fractional operators, Abstract Appl. Anal. 2014, Article ID 249091, 7(pp).

[25] Öğünmez, H. and Özkan, U.M.: Fractional quantum integral inequalities, J. Inequal. Appl., Volume 2011, Article ID 787939, 7 (pp).

[26] Özkan, U.M. and Yildirim, H.: Grüss type inequalities for double integrals on time scales, Comput. Math. Appl., 57 (2009), 436-444.

[27] Pachpatte, B.G.: On Grüss type integral inequalities, J. Inequa. Pure Appl. Math., 3 (1) (2002), 1-5.

[28] Pachpatte, B.G.: A note on Chebyshev-Grüss inequalities for differential equations, Tamsui Oxford Journal of Mathematical Sciences, 22(1), (2006), 29-36.
[29] Purohit, S.D. and Raina, R.K.: Chebyshev type inequalities for the Saigo fractional integrals and their q-analogues, J. Math. Inequal., 7(2) (2013), 239-249.

[30] Purohit, S.D. and Raina, R.K.: Certain fractional integral inequalities involving the Gauss hypergeometric function, Rev. Téc. Ing. Univ. Zulia, 37(2)(2014), In press.

[31] Purohit, S.D., U,car, F. and Yadav, R.K.: On fractional integral inequalities and their q-analogues, Revista Tecnocientifica URU, 6 (2014), In press.

[32] Wang, G., Agarwal, P. and Chand, M.: Certain Grss type inequalities involving the generalized fractional integral operator. Journal of Inequalities and Applications 2014, 2014:147.

[33] Tariboon, J., Ntouyas, S.K. and Sudsutad, W.: Some new Riemann-Liouville fractional integral inequalities, Int. J. Math. Math. Sci., 2014, Article ID 869434, 6 (pp).

[34] Yang, W.: On weighted q-Čebyšev-Grüss type inequalities, Comput. Math. Appl., 61 (2011), 1342-1347.

[35] Zhu, C., Yang, W. and Zhao, Q.: Some new fractional q-integral Gr"uss-type inequalities and other inequalities, J. Inequal. Appl., 2012 (2012), 299.

[36] Saigo, M.: A remark on integral operators involving the Gauss hypergeometric functions, Math. Rep. Kyushu Univ., 11 (1978) 135-143.

[37] Kiryakova, V.: Generalized Fractional Calculus and Applications (Pitman Res. Notes Math. Ser. 301), Longman Scientific \& Technical, Harlow, 1994. 